\title{
Programa de Reabilitação Cardíaca Home-based da Pessoa Transplantada ao Coração: RELATO DE CASO
}

\author{
PROGRAMA DE REHABILITACIÓN CARDÍACA DOMICILIARIO PARA LA PERSONA TRASPLANTADA DE CORAZÓN: \\ RELATO DE CASO
}

\section{Cardiac Rehabilitation Program for the Person Heart Transplanted at Home: Case Report}

DOI 10.33194/rper.2020.v3.s1.5.5771 | Submetido 29.04.2020 | Aprovado 26/10/2020

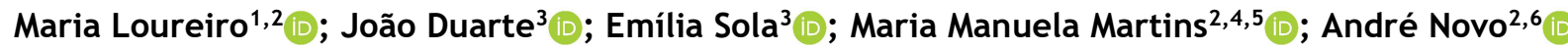 \\ 1 - Instituto de Ciências Biomédicas Abel Salazar, Centro Hospitalar e Universitário de Coimbra; 2 - NurseID-CINTESIS; \\ 3 - Centro Hospitalar e Universitário de Coimbra; 4 - Escola Superior de Enfermagem do Porto; \\ 5 - Instituto de Ciências Biomédicas Abel Salazar; 6 - Instituto Politécnico de Bragança
}

\section{RESUMO}

Contexto/Introdução: Os programas de reabilitação cardíaca (RC), nas suas diferentes fases, permitem ao enfermeiro especialista em enfermagem de reabilitação (EEER), ter uma intervenção privilegiada no acompanhamento e cuidado à pessoa transplantada ao coração, desde a fase pré-operatória, no período de recuperação pós-operatório mediato e tardio. Contribui para a promoção da autonomia e autocuidado da pessoa e sua família, na adaptação à nova condição de saúde e no aumento da sua qualidade de vida, intervindo nas diversas alterações psicológicas e fisiológicas decorrentes do transplante, sendo a reabilitação cardíaca uma opção terapêutica vantajosa neste contexto.

Objetivo: Analisar os ganhos em saúde potenciados pela Enfermagem de Reabilitação num Programa de Reabilitação cardíaca em domicílio.

Estratégias/metodologia: Relato de caso de abordagem mista, com a integração de 7 casos. Foram incluídas Pessoas transplantadas ao coração que nunca integraram programa de reabilitação cardíaca (PRC), manifestando alterações na capacidade funcional. Foram definidas como variáveis as seguintes características dos doentes: tempo de transplante, idade, género e medidas antropométricas. Foram avaliados parâmetros fisiológicos como frequência cardíaca (FC), tensão arterial (TA), perceção subjetiva de esforço (PSE) pela escala de Borg modificada e teste de marcha de 6 minutos, em 2 momentos de consulta de seguimento, com 3 meses de intervalo. No primeiro momento, após avaliação foi prescrito PRC em modelo Home-based. Foram efetuados contactos telefónicos e por correio eletrónico para perceber as dificuldades sentidas, bem como nível de cumprimento do programa. A mensuração de resultados foi efetuada num segundo momento de contacto.

Principais resultados: A integração no PRC proporcionou melhoria da capacidade de funcional, com $85,71 \%$ dos casos em estudo com incremento clinicamente significativo da distância no teste de marcha de 6 minutos, existindo uma melhoria da noção subjetiva de esforço em $57,14 \%$ das pessoas. Foram registadas melhorias do status dos diagnósticos de enfermagem comuns identificados, nomeadamente na Intolerância à atividade ausente; Autocuidado: atividade física não comprometido. Foi ainda registada melhoria do controlo de fatores de risco cardiovascular, como alimentação, stress, entre outros, todos os doentes alteraram parte do seu plano alimentar traduzindo-se em Adesão ao regime dietético não comprometida. No que se refere à qualidade de vida, $100 \%$ dos doentes apresentam discursos favoráveis à melhoria da qualidade de vida relacionada ao programa e acompanhamento. Não foram verificados eventos adversos durante o programa.

Conclusões: Neste estudo percebe-se que a participação em fase III de PRC é segura e traduz melhoria da capacidade funcional, maior capacidade de adesão ao regime dietético e controlo de fatores de risco cardiovascular, estando associada a discursos positivos da qualidade de vida.

Descritores: Transplante cardíaco, Reabilitação cardíaca; Enfermagem de Reabilitação o

\section{RESUMEN}

Contexto / Introducción: Los programas de rehabilitación cardíaca (CR), en sus diferentes fases, le permiten a la enfermera especialista de rehabilitación tener una intervención privilegiada en el monitoreo y cuidado de la persona trasplantada de corazón, desde la fase preoperatoria, en el período de recuperación mediato y tardío postoperatorio. Contribuye a la promoción de la autonomía y el autocuidado de la persona y su familia, adaptándose a la nueva condición de salud y aumentando su calidad de vida, interviniendo en los diversos cambios psicológicos y fisiológicos resultantes del trasplante, siendo la rehabilitación cardíaca una opción terapéutica ventajosa en este caso.

Propósito: Analizar los resultados en salud de la Enfermería de Rehabilitación en un programa de rehabilitación cardíaco a domicilio. 
Estrategias/metodología: Informe de casos de enfoque mixto, con la integración de 7 casos. Personas transplantadas de corazón que nunca integraron un programa de rehabilitación cardíaca (CRP), mostrando cambios en la capacidad funcional. Se definieron como variables las siguientes características de los pacientes: tiempo del trasplante, edad, sexo y medidas antropométricas. Los parámetros fisiológicos como el ritmo cardíaco (HR), la presión sanguínea (BP), la percepción subjetiva del estrés (PSE) por la escala de Borg modificada y una prueba de caminata de 6 minutos fueron evaluados en 2 visitas de seguimiento con un intervalo de 3 meses. En el primer momento, después de la evaluación, se prescribió un CRP. Se establecieron contactos telefónicos y por correo electrónico para comprender las dificultades experimentadas, así como el nivel de cumplimiento del programa. La medición de los resultados se realizó en un segundo momento de contacto.

Principales resultados: La integración en la PRC proporcionó una mejora en la capacidad funcional, con un 85,71\% de los casos estudiados con un aumento clínicamente significativo de la distancia en la prueba de caminata de 6 minutos, con una mejora en la noción subjetiva de esfuerzo en el 57,14\% de las personas. Se registraron mejoras en el estado de los diagnósticos de e enfermería más comunes identificados, a saber, en Intolerancia a la ausencia de actividad; Autocuidado: actividad física no comprometida. También hubo una mejora en el control de los factores de riesgo cardiovascular, como la nutrición, el estrés, entre otros, todos los pacientes cambiaron parte de su plan de dieta, lo que dio lugar a la adhesión a la dieta no comprometida. En cuanto a la calidad de vida, el $100 \%$ de los pacientes presentan discursos a favor de la mejora de la calidad de vida relacionados con el programa y el seguimiento. No se observaron eventos adversos durante el programa.

Conclusiones: En este estudio se observa que la participación en la fase III de la PRC es segura y se traduce en una mejora de la capacidad funcional, una mayor capacidad de adherencia al régimen dietético y en el control de los factores de riesgo cardiovascular, estando asociado a discursos positivos de calidad de vida.

Palabras clave: Trasplante de corazón, rehabilitación cardíaca; Enfermería de rehabilitación

\section{ABSTRACT}

Introduction: The cardiac rehabilitation programs (CRP), in its different phases, allow the rehabilitation nurse to have a privileged intervention in the monitoring and care of the person submitted to a heart transplant, from the preoperative phase, in the mediate recovery period and late postoperative. It contributes to the autonomy and selfcare of the person and his family, adapting to new health condition and increasing their quality of life, intervening in the various psychological and physiological changes resulting from transplant, with cardiac rehabilitation being an advantageous therapeutic option in this case.

Objective: To analyze the health outcomes of Rehabilitation Nursing in a Cardiac Rehabilitation Program homebased.

Strategies / methodology: Case report of mixed approach integrated 7 cases. There were included person submitted to a heart transplant who have never been part of a cardiac rehabilitation program (CRP), manifesting changes in functional capacity. The following patient characteristics were defined as variables: time of transplant, age, gender and anthropometric measurements. Physiological parameters such as heart rate (HR), blood pressure (BP), subjective perception of effort (PSE) were evaluated using the modified Borg scale and 6-minute walk test, in 2 moments of follow-up consultation, with a 3-months interval. At first, after evaluation, a PRC was prescribed in a Home-based model. Telephone and email contacts were made to understand the difficulties experienced, as well as the level of compliance with the program. The measurement of results was made in a second moment of contact.

Results: The integration in the CRP provided an improvement in functional capacity, with $85.71 \%$ of the cases of this study with clinically significant increase in the distance of the the 6-minutes walk test, with an improvement in the subjective notion of effort in $57.14 \%$ of subjects. There were improvements in the status of the common nursing diagnoses identified, such as Intolerance to activity; Self-care: physical activity not compromised. There was also an improvement in the control of cardiovascular risk factors, such as diet, stress, among others, all patients changed part of their diet plan, resulting in Adherence to the non-compromised diet.100\% of patients present speeches in favor of improving the quality of life related to the program and monitoring. There were no adverse events during the program.

Conclusions: In this study it is observed that the participation in phase III of CRP is safe and translates improvement of the functional capacity, greater capacity of adherence to the dietary regime and control of cardiovascular risk factors, being associated with positive discourses of quality of life.

Keywords: Heart transplantation, Cardiac rehabilitation; Rehabilitation Nursing

\section{INTRODUÇÃO}

A pessoa com insuficiência cardíaca refratária apresenta a condição terminal de diversas cardiopatias, caracterizada por agravamento do quadro clínico e mortalidade. Neste contexto, a terapêutica medicamentosa não consegue, em muitos casos, manter qualidade de vida adequada e sobrevida da pessoa, tendo o transplante cardíaco sido identificado 
como o tratamento para melhorar a qualidade ou prolongar a vida ${ }^{(1,2,3)}$.

O recurso a transplante cardíaco cresceu substancialmente nas últimas décadas, sendo agora realizados em 4000 doentes por ano em todo o mundo ${ }^{(4)}$,com sobrevida média pós-transplante entre 12 a $16 \operatorname{anos}^{(5)}$. Em Portugal, cerca de 950 pessoas foram submetidas a transplante do coração, nos cerca de 34 anos de início da atividade por Dr. Queirós de Melo.

O desenvolvimento da técnica cirúrgica, dos métodos de gestão medicamentosa, incluindo medicação imunossupressora, controlo de infeção e identificação e tratamento precoce da vasculopatia do aloenxerto, entre outras, resultaram em sobrevivência melhorada(6).

Apesar de ser atualmente uma técnica de sucesso, importa refletir sobre as limitações pré e pós transplante e formas de melhorar a qualidade de vida das pessoas. Atualmente, a técnica mais usada nos centros de transplantação é a bicaval (ortotópico), que consiste na desnervação do coração através da dissecação completa da aurícula direita e do septo interauricular, mantendo pequena porção da aurícula esquerda contendo as veias pulmonares. A desnervação cardíaca faz com que o controlo cardiorrespiratório (consumo máximo de $\mathrm{O}_{2}-\mathrm{VO}_{2 \text { máx) }}$ e hemodinâmico (frequência cardíaca, débito cardíaco e pressão arterial) sejam inicialmente dependentes do mecanismo de Frank-Starling (lei da pré carga dependente do retorno venoso), das catecolaminas circulantes (influenciam o inotropismo) e da pós-carga (através das anteriores acarretam variações da fração de ejeção), pela ausência da estimulação simpática e parassimpática e pelo barorreflexo ${ }^{(7,8)}$. Assim, as pessoas transplantadas cardíacas apresentam menores valores de $\mathrm{VO}_{2 \text { máx }}$ (cerca de $70-80 \%$ do que o valor previsto para idade em relação às pessoas saudáveis), elevados valores de frequência cardíaca, pressão arterial e resistência vascular em repouso, sendo que em exercício verifica-se um aumento débil da frequência cardíaca e pressão arterial, associado a aumento da resistência vascular.

Estas alterações aliadas aos efeitos da terapêutica imunossuressora geram alterações cardiorrespiratórias e hemodinâmicas imediatas e a longo prazo, podendo os transplantados cardíacos desenvolverem hipertensão arterial (95\%), dislipidémia (81\%), vasculopatia $(50 \%)$, insuficiência renal $(33 \%)$ e diabetes mellitus $(32 \%)^{(9)}$, sendo de suma importância a integração em programas de reabilitação nas suas diferentes fases, num continuum.

Apesar dos seus benefícios conhecidos, menos de $20 \%$ destas pessoas participam em programas de reabilitação cardíaca(12), em grande medida pela crença que o coração transplantado permanecia com incompetência cronotrópica. No entanto, existe agora ampla evidência de que o treino de exercício físico é bem tolerado e que este pode potenciar a reinervação e o controlo nervoso autónomo ${ }^{(3)}$, existindo um enfoque importante no exercício físico.

Os programas de reabilitação cardíaca em transplantação cardíaca devem incluir orientação nutricional, gestão do regime medicamentoso, intervenção psicológica no doente e família e plano de exercício/atividade física ${ }^{(13)}$.

Existe uma considerável variabilidade inerente ao carácter multifacetado e multiprofissional deste tipo de programas, quer quanto à existência dos diferentes componentes, ao tipo e duração das diferentes intervenções e mesmo quanto ao local onde o programa de reabilitação cardíaca é desenvolvido (centre-based [baseado em centro/unidade], home-based [baseado em casa], community-based [baseado na comunidade] $)^{(14)}$. Alguns autores quando analisam o melhor local para a realização de reabilitação em pessoas pós-transplante cardíaco, identificam que quer o programa hospitalar quer o ambulatório apresentam ganhos em saúde, contudo, com variáveis de resultado diferentes $^{(15)}$.

Neste contexto, e percebendo que o Enfermeiro de Reabilitação cuida de pessoas com necessidades especiais, ao longo do ciclo de vida, em todos os contextos da prática de cuidados, intervindo, entre outros, na função cardíaca e respiratória ${ }^{(16)}$, facilitando o processo de transição, deverá ser um dos elementos integrantes da equipa de reabilitação cardíaca. Os programas de reabilitação cardíaca $(R C)$, nas suas diferentes fases, permitem ao enfermeiro especialista em enfermagem de reabilitação (EEER) ter uma intervenção privilegiada no acompanhamento e cuidado à pessoa com doença cardíaca, desde a fase aguda da doença ao período de recuperação, capacitando-a para a gestão do binómio saúde/doença, contribuindo para a promoção da autonomia e autocuidado da pessoa e sua família, na adaptação à nova condição de saúde e no aumento da sua qualidade de vida ${ }^{(17)}$.

Assim, tentar-se-á responder à seguinte questão: Será que um programa de RC aplicado a pessoas transplantados ao coração por enfermeiros de reabilitação, em contexto home based, apresenta ganhos em saúde?

\section{MÉTODO}

Os relatos de caso foram elaborados com base nas orientações da CAse REport (CARE) ${ }^{(17)}$, uma vez que estas permitem a conceção de uma estrutura de estudo de caso mais lógica e clara, apresentado uma proposta de organização do mesmo em vários itens de relevo. Foram cumpridos os itens sendo feita a adaptação necessária aos casos em questão.

Os dados apresentados referem-se a sete pessoas transplantadas cardíacas de um Centro de Transplantação do país. Os critérios de inclusão no estudo foram 1) idade superior a 18 anos; 2) transplante ao coração há mais de 2 meses, 3) nunca terem integrado programa de RC, 4) capacidade para fornecer consentimento informado.

Em consenso de equipa avaliou-se para que doentes era segura a prescrição de um programa de RC home based, assim como a capacidade do doente e se existia o apoio familiar necessário. 
Assim, aos 7 participantes foi efetuada uma avaliação inicial antropométrica, sinais vitais, oximetria periférica, noção subjetiva de esforço (Escala de Borg modificada) e teste de caminhada de 6 minutos (com telemetria aplicada durante o teste). Após esta avaliação foram construídos os diagnósticos de enfermagem de reabilitação inerentes.

Foi aplicado o protocolo Physical Activity4HTxHome (PA4HTXH), que integra 4 modalidades de treino: Exercício de aquecimento, Treino aeróbio, Treino de força e Alongamentos no final (conforme imagem 1). A prescrição foi baseada no conceito FITT-P (Frequência, Intensidade, Tipo, Tempo e Progressão), adaptada às características individuais dos participantes, atividade laboral e disponibilidade de tempo. A prescrição do programa foi efetuada numa folha com imagens alusivas ao tipo de treino, tempo, carga e foi explicado que deveriam avaliar sinais vitais antes e após o treino, os sinais de alarme para suspender o exercício ou não fazer progressão do treino. Semanalmente, os doentes progrediriam de acordo com a sua tolerância. Foram identificados fatores de risco cardiovascular modificáveis e efetuada intervenção no sentido da sua correção, durante o período de 3 meses. 0 modelo do programa foi Home based, sendo o cuidador de referência envolvido como parceiro de cuidados no sentido de incentivar o cumprimento do plano e envolver-se na modificação de alguns comportamentos de risco identificados. De forma a validar o cumprimento do programa, foram efetuados contactos telefónicos e eletrónicos com doentes e cuidadores de forma a percecionar dificuldades, algum tipo de complicação, resposta ao exercício físico necessidades de ajuste do programa. Foram avaliados os mesmos parâmetros iniciais na consulta de seguimento com 3 meses de diferença.

No dia da 2. a avaliação foi entregue uma folha aos doentes com uma questão aberta, de forma a avaliar a sua perspetiva de qualidade de vida após o PRC: "Como alterou a sua vida a integração no Programa de Reabilitação Cardíaca?"

Foram cumpridas todas as normas de proteção na recolha e tratamento dos dados e obtido consentimento livre e esclarecido.

Para a descrição dos dados recorreu-se ao programa Microsoft Excel.

Identificação de fatores de risco cardiovascular modificáveis e intervenção individualizada, como: Obesidade, Alterações do perímetro abdominal, HTA, Diabetes, entre outros

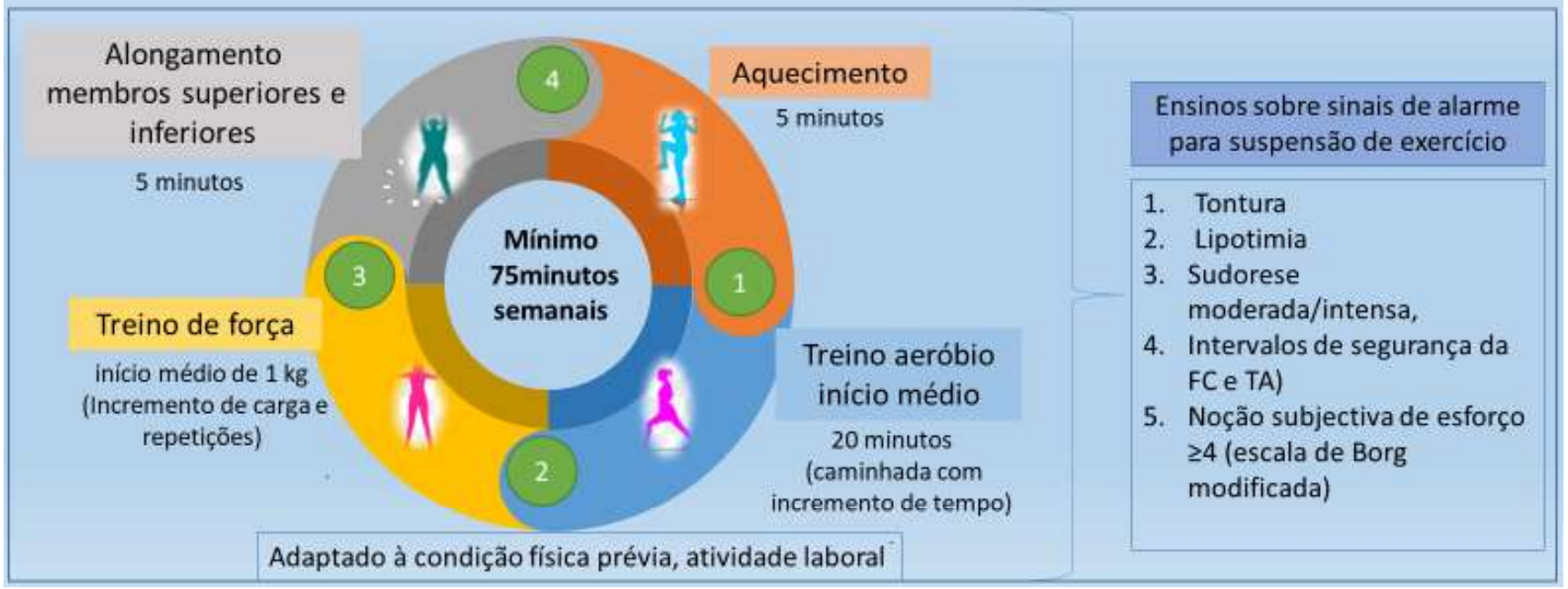

Figura 1- Programa Physical Activity4HeartTransplant Home-based (PA4HTxH)

\section{RESULTADOS}

Foram analisados 7 casos, 71,4\% Homens e 28,6\% Mulheres, com, em média, 58 anos de idade e 6 anos de transplante. Todos são casados ou vivem em União de Facto, sendo o cônjuge o seu cuidador informal identificado. Apresentam polipatologia (sobretudo doenças metabólicas, diabetes, hipertiroidismo, ...), sendo que $85,71 \%$ tinham pelo menos um dos fatores de risco cardiovascular modificáveis e nenhum tinha integração prévia em Programa de Reabilitação Cardíaca. Todos se referem como independentes nos seus autocuidados, desempenhando a sua função laboral sem dificuldades, mas com intolerância à atividade. Os motivos de doença cardíaca prévia são variados, contudo com o novo órgão a intervenção da
RC deverá ser também de gestão dos fatores de risco da doença cardiovascular.

Durante a avaliação foram construídos os diagnósticos de enfermagem em linguagem classificada, sendo que todos manifestavam conhecimento demonstrado sobre gestão do regime terapêutico medicamentoso, mas conhecimento não demonstrado sobre gestão do regime terapêutico: exercício, com intolerância à atividade e autocuidado atividade física: comprometido, não apresentavam comportamento de adesão ao adequado regime alimentar, apesar de conhecimento. Foi também identificado o diagnóstico Gestão dos fatores de risco cardiovascular comprometido, ainda que não contemplada na parametrização nacional. 
Avaliação de Enfermagem de Reabilitação

Relativamente aos dados antropométricos iniciais relevantes para a determinação do risco cardiovascular inicial encontram-se infraescritos na Tabela 1.

Utilizando os dados obtidos nas avaliações da Noção subjetiva de esforço, Teste de marcha de 6 minutos, sinais vitais, oximetria periférica, avaliação antropométrica, foram identificados os seguintes diagnósticos de enfermagem de reabilitação:
- Intolerância à atividade, presente

- Autocuidado: atividade física comprometido

- Exercício (integrado no autocuidado: atividade física)

- Adesão do regime terapêutico dietético comprometido

- Gestão dos fatores de risco cardiovascular

Todos os diagnósticos encontravam-se resolvidos no final da intervenção.

\begin{tabular}{|c|c|c|c|c|c|c|c|}
\hline Caso & Gén. & $\begin{array}{l}\text { Idade } \\
\text { (anos) }\end{array}$ & $\begin{array}{l}\text { Tempo de transplante } \\
\text { (anos) }\end{array}$ & $\begin{array}{l}\text { Peso } \\
(\mathrm{Kg})\end{array}$ & $\begin{array}{l}\text { Altura } \\
\text { (m) }\end{array}$ & IMC & $\begin{array}{l}\text { Perímetro abdominal } \\
(\mathrm{cm})\end{array}$ \\
\hline 1 & $\mathrm{~F}$ & 39 & 11 & 63 & 1,60 & 24.61 & 95 \\
\hline 2 & $M$ & 59 & 0,25 & 67 & 1,68 & 23.74 & 99,5 \\
\hline 3 & $F$ & 56 & 13 & 78 & 1,57 & 31.64 & 104 \\
\hline 4 & $M$ & 63 & 0,5 & 80 & 1,76 & 25.83 & 114 \\
\hline 5 & $M$ & 70 & 3 & 73 & 1,58 & 29.24 & 102 \\
\hline 6 & $M$ & 53 & 4 & 80 & 1,70 & 27.68 & 106 \\
\hline 7 & $M$ & 64 & 4,5 & 75 & 1,70 & 25.95 & 99,5 \\
\hline
\end{tabular}

Legenda: F- Feminino; M-Masculino, IMC- Índice de Massa corporal

Tabela 1-Dados antropométricos

Caso Gén. Diferença peso Diferença per. abdominal Média da diferença \% redução do per. abdominal

\begin{tabular}{|c|c|c|c|}
\hline 1 & $F$ & $(+) 2 \mathrm{Kg}$ & $(+) 3 \mathrm{~cm}$ \\
\hline 2 & $M$ & $(+) 3 \mathrm{~kg}$ & $(+) 1 \mathrm{~cm}$ \\
\hline 3 & $F$ & $(-) 4 \mathrm{~kg}$ & $(-) 10 \mathrm{~cm}$ \\
\hline 4 & $M$ & $(+) 4 \mathrm{~kg}$ & $(-) 5 \mathrm{~cm}$ \\
\hline 5 & $M$ & $(-) 3 \mathrm{Kg}$ & $(-) 1 \mathrm{~cm}$ \\
\hline 6 & $M$ & $(+) 3 \mathrm{~kg}$ & $(-) 3 \mathrm{~cm}$ \\
\hline 7 & $M$ & $(+) 1 \mathrm{~kg}$ & 0 \\
\hline
\end{tabular}

Tabela 2 - Dados antropométricos: diferenças entre avaliações

\begin{tabular}{|c|c|c|c|c|c|c|c|c|}
\hline Caso & $\begin{array}{l}\text { Distância } \\
\text { inicial }(m)\end{array}$ & $\begin{array}{l}\text { Distância } \\
\text { final (m) }\end{array}$ & $\begin{array}{l}\text { Diferença } \\
\qquad(\mathrm{m})\end{array}$ & $\begin{array}{l}\text { Incremento } \\
\text { médio }(\mathrm{m})\end{array}$ & $\begin{array}{c}\% \text { doentes com } \\
\text { incremento da } \\
\text { capacidade funcional }\end{array}$ & $\begin{array}{l}\text { Borg } \\
1 .^{\mathrm{a}}\end{array}$ & $\begin{array}{l}\text { Borg } \\
2 .^{\mathrm{a}}\end{array}$ & $\begin{array}{c}\% \text { doentes com } \\
\text { melhoria da noção } \\
\text { subjetiva de esforço }\end{array}$ \\
\hline 1 & 322 & 336 & +14 & \multirow{7}{*}{152,285} & \multirow{7}{*}{$85,71 \%$} & 5 & 3 & \multirow{7}{*}{$57,14 \%$} \\
\hline 2 & 322 & 441 & +119 & & & 2 & 2 & \\
\hline 3 & 248 & 364 & +116 & & & 4 & 3 & \\
\hline 4 & 392 & 995 & +603 & & & 0 & 0 & \\
\hline 5 & 278 & 336 & +58 & & & 1 & 0 & \\
\hline 6 & 476 & 560 & +84 & & & 0 & 0 & \\
\hline 7 & 320 & 392 & +72 & & & 4 & 2 & \\
\hline
\end{tabular}

Tabela 3 - Análise do Teste de Marcha de 6 minutos e Noção subjetiva de esforço entre avaliações

\begin{tabular}{|c|c|c|c|c|c|c|}
\hline Caso & $\begin{array}{l}\text { TA 1. } \\
(\mathrm{mmHg})\end{array}$ & $\begin{array}{l}\text { TA 2. }{ }^{\mathrm{a}} \\
(\mathrm{mmHg})\end{array}$ & $\begin{array}{l}\text { FC } 1 .^{\text {a }} \text { repouso } \\
\text { (bat } / \mathrm{min})\end{array}$ & $\begin{array}{l}\text { FC } 2 .^{\text {a }} \text { repouso } \\
\text { (bat } / \mathrm{min})\end{array}$ & $\begin{array}{c}\text { FC 1. }{ }^{\text {a }} \text { pós exercício } \\
\text { (bat } / \mathrm{min})\end{array}$ & $\begin{array}{c}\text { FC } 2 .{ }^{a} \text { pós exercício } \\
\text { (bat } / \mathrm{min} \text { ) }\end{array}$ \\
\hline 1 & $115 / 72$ & $137 / 75$ & 92 & 72 & 92 & 78 \\
\hline 2 & $123 / 83$ & $134 / 82$ & 90 & 84 & 101 & 94 \\
\hline 3 & $121 / 63$ & $113 / 67$ & 80 & 60 & 82 & 64 \\
\hline 4 & $135 / 91$ & $124 / 91$ & 94 & 101 & 103 & 100 \\
\hline 5 & $144 / 85$ & $120 / 70$ & 90 & 89 & 90 & 92 \\
\hline 6 & $129 / 73$ & $117 / 73$ & 70 & 68 & 69 & 75 \\
\hline 7 & $152 / 71$ & $127 / 76$ & 103 & 84 & 98 & 97 \\
\hline
\end{tabular}

Tabela 4 - Valores de Tensão arterial, Frequência Cardíaca em repouso e Frequência Cardíaca pós exercício. 
Em relação à qualidade de vida avaliada aqui pela noção subjetiva, na análise de conteúdo efetuada às respostas identificam-se apenas aspetos de melhoria da qualidade de vida com o PRC. Sendo assim, foram identificadas 4 categorias conforme o quadro 1:

\begin{tabular}{|l|l|}
\hline \multicolumn{1}{|c|}{ Categorias } & \multicolumn{1}{|c}{ Unidades de Registo } \\
\hline Autoestima & “agora visto roupa que já não me servia há anos" (C3) \\
\hline Humor & $\begin{array}{l}\text { "Faço coisas que não fazia antes, sinto-me feliz por isso" (C2), "Sinto um "folego" diferente, } \\
\text { mesmo quando recebi o coração não tinha esta sensação de bem-estar" (C7); "Voltei a lide } \\
\text { agrícola. Tinha tantas saudades" (C4) }\end{array}$ \\
\hline Vida familiar & $\begin{array}{l}\text { "Acompanho melhor a minha filha a brincar" (C5) "Acompanho a minha mãe nas caminhadas dela } \\
\text {..antes não o fazia" (C3); }\end{array}$ \\
\hline $\begin{array}{l}\text { Capacidade } \\
\text { física }\end{array}$ & $\begin{array}{l}\text { "Comecei a ir a pé para o trabalho." (C1) “Voltei à lide agrícola." (C4), "Faço caminhadas como } \\
\text { antigamente, agora sem desistir a meio" (C6) }\end{array}$ \\
\hline
\end{tabular}

Quadro 1 - Qualidade de Vida-Categorias

\section{DISCUSSÃO}

A análise conjunta de dados antropométricos, hemodinâmicos e funcionais, revela-se fundamental para compreender o impacto de um programa de reabilitação cardíaca efetuado por enfermeiro de reabilitação no contexto da pessoa transplantada ao coração. Dos estudos analisados sobre os efeitos da reabilitação cardíaca neste contexto comparam-se com os resultados genéricos das pessoas estudadas, percebendo-se que o PRC apresentou diversos dos efeitos descritos na literatura, conforme Quadro 2.

\section{Os efeitos da reabilitação cardíaca em} transplantação ${ }^{(7,11,20)}$ :

- Redução dos sintomas de depressão e ansiedade

- Redução da ativação neuro-hormonal do BNP e ANP

- $\uparrow$ do VO2máx

- $\downarrow$ da FC em repouso

- $\uparrow$ da FC em exercício

- Melhoria da fração de ejeção

- Melhoria da incompetência cronotrópica

- Melhoria da vascularização arteriovenosa

- Redução da gordura corporal e melhoria de perfil lipídico

- Diminuição das pressões arteriais sistólica e diastólica em repouso e exercício submáximo

- Melhoria do nível de dor

- Melhoria da densidade óssea e diminuição da sarcopenia

- Controlo dos fatores de risco CV

- Melhoria da qualidade de vida

- Melhoria da capacidade funcional

- Redução dos reinternamentos

- Redução de eventos CVs

Quadro 2 - Efeito do Programa de Reabilitação Cardíaca

\section{Capacidade Funcional/Intolerância à Atividade}

A habilidade da marcha é um indicador confiável de capacidade funcional, sendo que o seu aumento de pelo menos 48 metros $^{(22)}$ representa a diferença mínima clinicamente significativa de benefício físico após um PRC. $85,71 \%$ dos casos em estudo apresentaram ganhos da capacidade funcional, com incremento acima dos 48 metros, o que reitera o descrito por Kawauchi, et.al (2013). Relativamente ao único doente que não apresentou esse ganho, verificou-se junto do cuidador

\section{Resultados das pessoas estudadas}

- Melhoria do Humor

- Diminuição da FC em repouso

- Diminuição da FC em exercício

- Redução do perímetro abdominal

- Diminuição das pressões arteriais sistólica e diastólica em repouso e exercício submáximo

- Melhoria no controlo de fatores de risco

- Discursos positivos em relação à qualidade de vida

- Melhoria da capacidade funcional

- Melhoria da noção subjetiva de esforço

- Sem registo de nenhum evento cardiovascular

que o doente não cumpria de forma sistematizada o programa prescrito, o que pode justificar este resultado.

Percebe-se uma relação inversamente proporcional e significativa da melhoria da capacidade funcional e do perímetro abdominal, tal como evidenciado por Forestieri et al (2016) no seu estudo.

No que se refere à Intolerância a atividade, houve $85,71 \%$ de doentes que apresentaram melhoria da sua noção subjetiva de esforço, o que vai ao encontro do 
descrito por Oliveira et al. (2016), que descreve que um programa de RC fase III reduz a noção subjetiva de esforço (pela Escala de Borg).

\section{Fatores de Risco}

Analisando os valores de referência considerados para a população portuguesa, percebe-se que $85,71 \%$ apresentam fator de risco para desenvolver doenças cardiovascular (19,20), nomeadamente obesidade, alterações do perímetro abdominal, comorbilidades (HTA, Diabetes).

Cinco dos doentes apresentam pré-obesidade (valor igual ou superior a 25) e 2 obesidade, sendo que 2 apresentam obesidade, no início do PRC.

Também no que se refere ao perímetro abdominal (como indicador de saúde), pelo valor que se apresenta, todos têm risco "Muito Aumentado" de complicações metabólicas, considerando os valores da Organização Mundial de Saúde:

- sem risco - mulheres < $80 \mathrm{~cm}$ e homens < $94 \mathrm{~cm}$;

- risco aumentado - mulheres $80 \mathrm{~cm}<P C \leq 88 \mathrm{~cm}$ e homens $94 \mathrm{~cm}<\mathrm{PC} \leq 102 \mathrm{~cm}$;

- risco substancialmente aumentando - mulheres $>88$ $\mathrm{cm}$ e homens $>102 \mathrm{~cm}$.

Contudo, a integração em PRC levou a que $57,14 \%$ dos doentes reduzissem perímetro abdominal; estes resultados vão ao encontro do sugerido no estudo de Uithoven et al (2020).

A reter que nenhum dos doentes teve introdução medicamentosa inaugural, sendo que a única variável presento foi o PRC.

Em relação às comorbilidades, houve melhoria num dos doentes que era diabético, nos valores em jejum da glicémia, e na necessidade de suspensão da insulina prescrita há 2 anos. Apesar de ter existido aumento ponderal com incremento do IMC, a redução do perímetro abdominal associada, em razão inversa, pode ser justificado pelo aumento da massa muscular, conduz à necessidade de estudos com mais variáveis relacionadas com os valores antropométricos.

No que se refere à hemodinâmica, a maioria dos casos analisados apresentaram redução da frequência cardíaca, o que podemos inferir tratar-se de melhoria da morfologia ou estrutura do ventrículo esquerdo, assim como determinadas respostas fisiológicas como o aumento do volume ejetado por sístole, melhoria da contratilidade miocárdica e aumento do volume sanguíneo tal como referido no estudo de Paschoal et al. (2019).

No que se refere aos valores de tensão arterial, houve também reduções clinicamente significativas na maioria dos casos, o permite a redução de um dos fatores de risco cardiovascular.

Os resultados de redução de fatores de risco modificáveis vão ao encontro do estudo de Uithoven et al (2020), que descreve que a RC foi associada à prevenção de fatores de risco e deve ser vista como uma ferramenta fundamental nas estratégias de tratamento pós-transplante.

A implementação do PA4HTxH permitiu que em 85,71\% dos casos tenha apresentado ganho de capacidade funcional. A alteração de determinados comportamentos (regime dietético sobretudo) levaram à resolução dos diagnósticos de enfermagem de reabilitação identificados, com melhoria da gestão do regime terapêutico como evidenciado por Ciolac et al. (2014).

\section{Qualidade de Vida}

A questão colocada relativa à relação da qualidade de vida identificou discursos positivos com a integração no programa de reabilitação, o que vai ao encontro do descrito por Uithoven et al. (2020), que descrevem que os benefícios da participação de RC após o transplante cardíaco são substanciais e generalizados e influenciam a qualidade de vida muito além do período inicial de atendimento.

$\mathrm{Na}$ análise dos discursos, identifica-se o Humor como uma das categorias identificadas, o que vai ao encontro do estudo de Chen et. al. (2010) que referencia diminuição de alterações psíquicas decorrentes do incremento do exercício.

\section{Limitações do estudo}

O facto de ser uma intervenção não supervisionada limita a total garantia do cumprimento do plano de treino de exercício físico e das alterações comportamentais prescritas.

\section{CONCLUSÃO}

Este estudo permitiu evidenciar o papel do enfermeiro de reabilitação no acompanhamento e follow-up no programa de reabilitação cardíaca à pessoa transplantada, existindo ganhos em saúde quando a pessoa transplantada ao coração é integrada num programa de RC, fase III.

Torna-se fundamental perceber a diferença (se existente) entre os programas supervisionados ou não supervisionados em Home based.

Paralelamente será também importante perceber quais as diferenças de resultado quando o doente se encontra integrado em PRC desde a fase pré-operatória.

\section{REFERÊNCIAS BIBLIOGRÁFICAS}

1. Lund LH, Khush KK, Cherikh WS, Goldfarb S, Kucheryavaya AY, Levvey BJ, et al. The Registry of the International Society for Heart and Lung Transplantation: Thirty-fourth Adult Heart Transplantation Report-2017; Focus Theme: Allograft ischemic time. J Hear Lung Transplant. 2017 Oct;36(10):1037-46. Available from: https://doi.org/10.1016/j.healun.2017.07.019

2. Nygaard S, Christensen AH, Rolid K, Nytrøen K, Gullestad L, Fiane $A$, et al. Autonomic cardiovascular control changes in recent heart transplant recipients lead to physiological limitations in response to orthostatic challenge and isometric exercise. Eur J Appl Physiol. 2019 Aug 12; Available from: https://doi.org/10.1007/s00421-019-04207-5 
3. Anderson L, Nguyen TT, Dall CH, Burgess L, Bridges C, Taylor RS. Exercise-based cardiac rehabilitation in heart transplant recipients. Cochrane Database Syst Rev. 2017 Apr 4; Available from: https: //doi.org/10.1002/14651858.CD012264.pub2

4. Rosenbaum AN, Kremers WK, Schirger JA, Thomas RJ, Squires RW, Allison TG, et al. Association Between Early Cardiac Rehabilitation and Long-term Survival in Cardiac Transplant Recipients. Mayo Clin Proc. 2016 Feb;91(2):149-56. Available from: https://doi.org/10.1016/j.mayocp.2015.12.002

5. International Society of Heart and Lung Transplantation. International Thoracic Organ Transplant (TTX) Registry Data Slides [Internet]. 2018.

6. Söderlund C, Rådegran G. Immunosuppressive therapies after heart transplantation--The balance between under- and overimmunosuppression. Transplant Rev (Orlando). 2015;29(3):181189. doi:10.1016/j.trre.2015.02.005

7. Loureiro MFS. Reabilitação e transplante cardíaco: revisão sistemática da literatura. 2015 [cited 2019 Oct 18]

8. Awad M, Czer LS, Hou M, et al. Early Denervation and Later Reinnervation of the Heart Following Cardiac Transplantation: A Review. J Am Heart Assoc. 2016;5(11):e004070. Published 2016 Nov 1. doi:10.1161/JAHA.116.004070

9. Perrier-Melo RJ, dos Santos Figueira FAM, Guimarães GV, da Cunha Costa M. Treinamento Intervalado de Alta Intensidade em Transplantados de Coração: Uma Revisão Sistemática com MetaAnálise. Arq Bras Cardiol. 2018;110(2):188-94.

10. ACSM. ACSM's guideline for exercise testing and prescription. Tenth. American College of Sports Medicine; 2018. 501 p.

11. Bachmann JM, Shah AS, Duncan MS, Greevy RA, Graves AJ, Ni S, et al. Cardiac rehabilitation and readmissions after heart transplantation. J Hear Lung Transplant. 2018 Apr;37(4):467-76. Available from: https://doi.org/10.1016/j.healun.2017.05.017

12. Fogaça D, Goés GG de, Fuhro MI, Mariante P, Frasson R, Monteiro $M B$, et al. $O$ papel da reabilitação física após o transplante cardíaco: uma revisão da literatura. EFDeportes. 2012;(171)

13. Rocha JAGM da. Efectividade de um programa de reabilitação cardíaca [Internet]. Dissertação de candidatura ao grau de Mestre em Epidemiologia. Faculdade de Medicina. Universidade do Porto; 2010.

14. Karapolat H, Eyigor S, Zoghi M, Yagdi T, Nalbantgil S, Durmaz B, et al. Effects of cardiac rehabilitation program on exercise capacity and chronotropic variables in patients with orthotopic heart transplant. Clin Res Cardiol. 2008 Jul 3;97(7):449-56. Available from: https://doi.org/10.1007/s00392-008-0648-7

15. Regulamento $n .^{\circ} 392 / 2019$ Regulamento das competências específicas do enfermeiro especialista em Enfermagem de

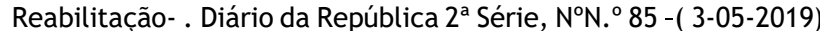
13565-13568

16. Regulamento $n^{\circ} 350 / 2015$ de 22 de Junho (2015). Regulamento dos padrões de qualidade dos cuidados especializados em

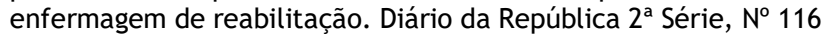
(22-06-2015) 16655-16660.

17. Riley, D. S., Barber, M. S., Kienle, G. S., Aronson, J. K., von Schoen-Angerer, T., Tugwell, P., Gagnier, J. J. (2017). CARE guidelines for case reports: explanation and elaboration document. J Clin Epidemiol, 89, 218-235. doi:10.1016/j.jclinepi.2017.04.026

18. Borracci, R. A., Ingino, C. A., \& Miranda, J. M. (2018). Association of body mass index with short-term outcomes after cardiac surgery: Retrospective study and meta-analysis. Med, 78(3), 171 179.

19. Organização Mundial da Saúde (OMS). (2001) Waist circunference and waist-hip ratio: report of a WHO expert consultation. Geneve: Organização Mundial da Saúde;. 8

20. Direção-Geral da Saúde (DGS). Orientação da Direção-Geral da Saúde: Avaliação Antropométrica no Adulto. Lisboa: DireçãoGeral da Saúde; 2013. 1-9.

21. Uithoven, K. E., Smith, J. R., Medina-Inojosa, J. R., Squires, R. W., \& Olson, T. P. (2020). The Role of Cardiac Rehabilitation in Reducing Major Adverse Cardiac Events in Heart Transplant Patients. J Card https://doi.org/10.1016/j.cardfail.2020.01.011

22. Kawauchi, e. a. (2013). Randomized and comparative study between two intra-hospital exercise programs for heart transplant patient. Rev Bras Cir Cardiovasc, 28(3), pp. 338- 346.
23. Forestieri, P., Guizilini, S., Peres, M., Bublitz, C., Bolzan, D. W., Rocco, I. S., Santos, V. B., Moreira, R. S., Breda, J. R., de Almeida, D. R., Carvalho, A. C., Arena, R., \& Gomes, W. J. (2016). A Cycle Ergometer Exercise Program Improves Exercise Capacity and Inspiratory Muscle Function in Hospitalized Patients Awaiting Heart Transplantation: a Pilot Study. Braz J Cardiovasc Surg, 31(5), 389-395. https://doi.org/10.5935/1678$\underline{9741.20160078}$

24. Ciolac, e. a. (2014). Prescribing and Regulating Exercise with RPE after Heart Transplant: A Pilot Study. Medicine \& Science in Sports \& Exercise

25. Oliveira, A.; Costa, D., Teodoro, E. ; Pereira, W.; Miranda, V., (2016) Reabilitação cardíaca fase III em pacientes submetidos ao transplante cardíaco in Ver Bras Fisiol Exerc - ano 2016 - volume 15 - número 2. DOI: http://dx.doi.org/10.33233/rbfe.v15i2.147

26. Chen, e. a. (2010). Mediating effect of symptom severity on the relationship between self-efficacy for exercise and depressio. $J$ Clin Nurs, 20, pp. 294-296 